\title{
Infantile Sandhoff's disease
}

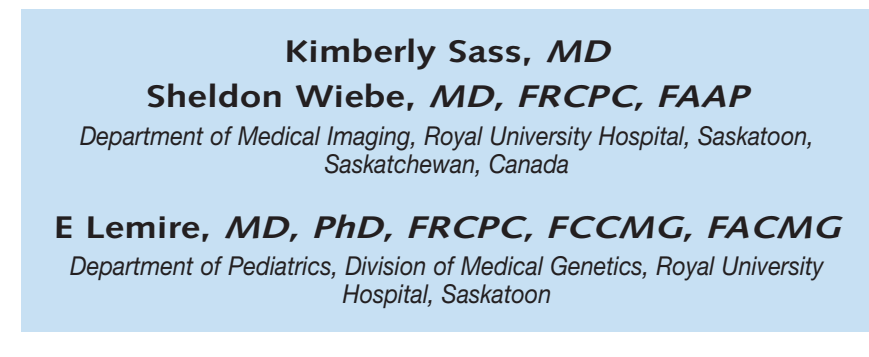

\section{Abstract}

Sandhoff's disease is a rare autosomal recessive disorder of sphingolipid metabolism that results from deficiency of the lysosomal enzymes, $\beta$-hexosaminidase $A$ and $B$. The resultant accumulation of $\mathrm{GM}_{2}$ ganglioside within both grey matter nuclei and myelin sheaths of the white matter results in eventual severe neuronal dysfunction and neurodegeneration. Disease progression is rapid, resulting in early death. Currently, there is no curative treatment, with therapy remaining primarily supportive.

This case report is of a 13-month-old aboriginal Canadian boy who was referred for further investigations related to global developmental delay and loss of developmental milestones, at which time the diagnosis was discovered.

\section{Clinical presentation}

A 13-month-old Canadian aboriginal boy presented with global developmental delay and loss of developmental milestones. He was born at 38 weeks to nonconsanguinous parents after an uncomplicated pregnancy. He experienced normal development until the age of 3 months, at which time-delayed motor skills, followed by episodes of myoclonic jerks, were observed. Verbal and social development was normal until approximately 6 months of age.

Physical examination was remarkable for increased head circumference relative to weight, with frontal bossing and hypotonic, nondysmorphic facies. No organomegaly was present. Neurological examination revealed central hypotonia with head lag and symmetric spasticity involving all four limbs. Deep tendon reflexes were normal, with the exception of ankle clonus and upgoing plantar reflexes. Bilateral macular cherry red spots were visualised, and visual evoked responses were absent. Further investigations including routine bloodwork and metabolic studies were normal. An EEG showed excessive slowing of delta frequencies associated with drowsiness. Plasma hexosaminidase levels were significantly decreased.

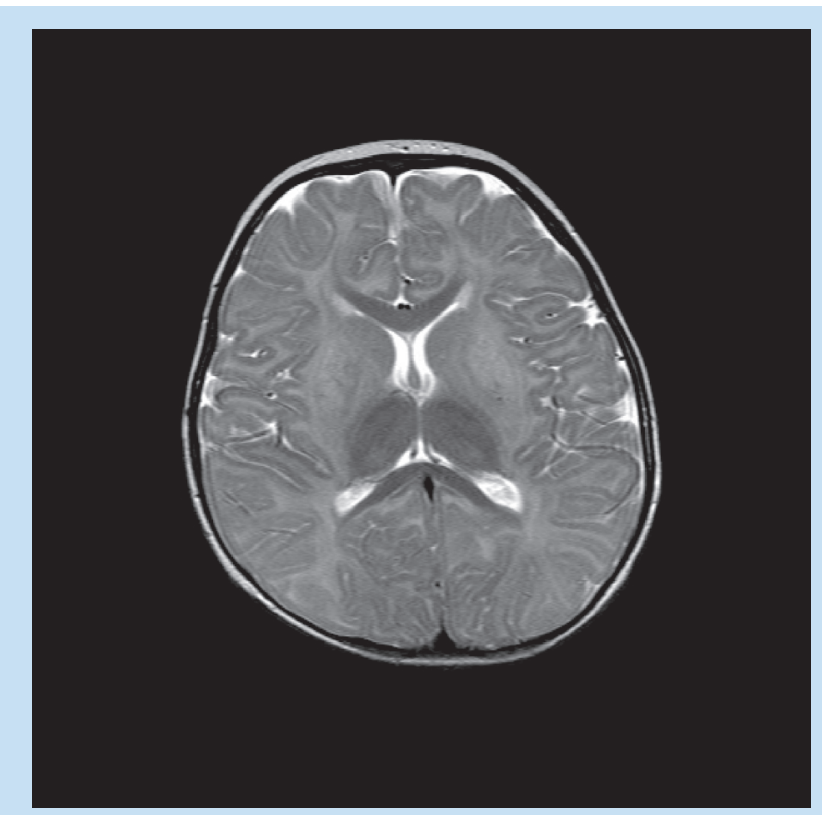

Fig. 1. Axial T2WI at the thalamic level demonstrating bilateral diffusely hypointense thalami, hyperintense speckled putamina, and delayed myelination.

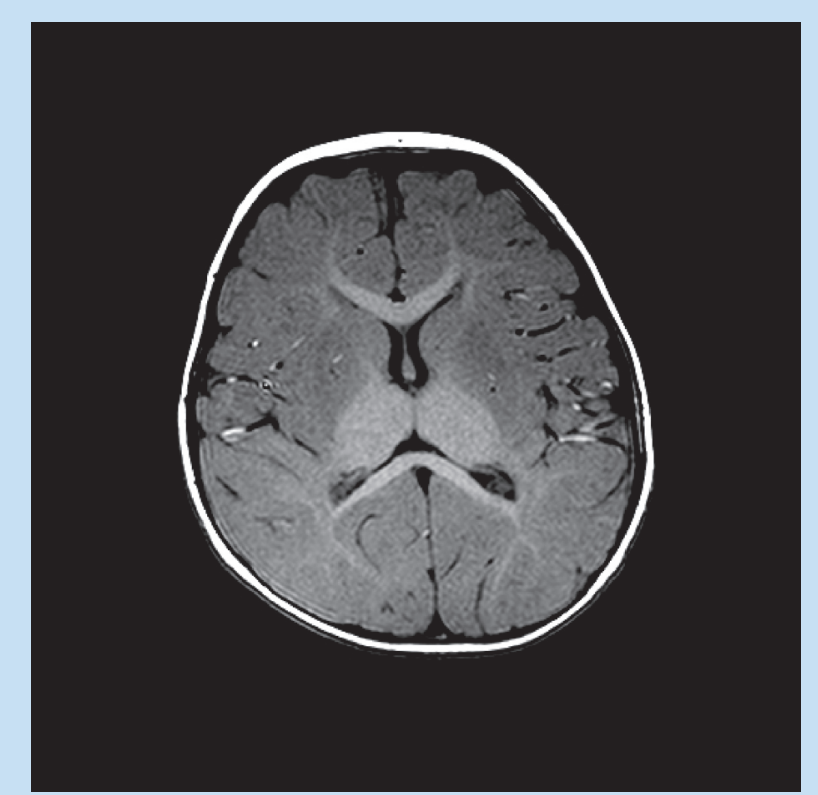

Fig. 2. Axial T1WI at the thalamic level demonstrating diffuse thalamic hyperintensity. 


\section{Diagnosis}

The diagnosis was infantile onset Sandhoff's disease.

\section{Radiographic findings}

Bilateral, diffuse, T1 hyper-, T2 (Figs 1 and 2) and FLAIR hypointense (not shown) thalami were seen. Hyperintense, speckledappearing putamina on $\mathrm{T} 2 \mathrm{~W}$ images containing punctate $\mathrm{T} 2$ and FLAIR hypointense foci are demonstrated in Fig. 1. Additional MRI findings include a delayed myelination pattern for age, with no visible areas of diffusion restriction.

Single voxel stimulated echo acquisition mode (STEAM) acquisition of the right lentiform nucleus for magnetic resonance spectroscopy revealed diminished $\mathrm{N}$-acetylaspartate (NAA), suggestive of neuronal loss/dysfunction (Fig. 4). Multivoxel acquisition through deep grey matter structures revealed diffusely diminished NAA, most notable within the thalami (not shown). Choline levels were found to be elevated which, while a nonspecific finding, may relate to dysmyelination. A few, small, nonspecific lactate peaks were also seen.

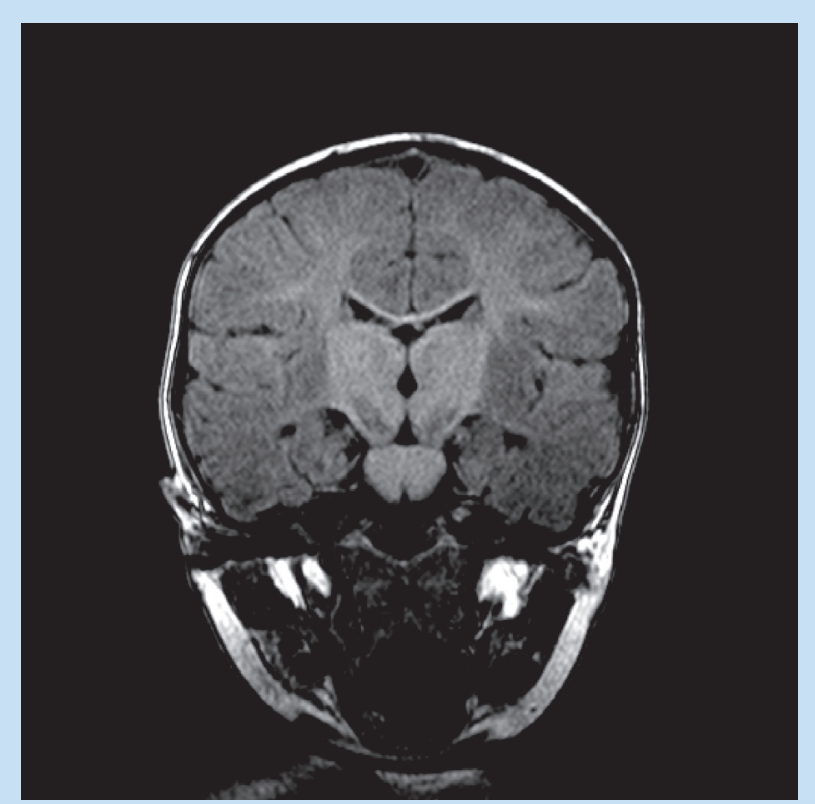

Fig. 3. Coronal MPRAGE demonstrating diffuse thalamic hyperintensity.

\section{Discussion}

Sandhoff's disease is a rare autosomal recessive disorder of sphingolipid metabolism, with an incidence of 1 in 384000 live births, ${ }^{1}$ related to a genetic deficiency of the enzyme $\beta$ hexosaminidase. Two catalytically active forms of this enzyme exist: $\beta$-hexosaminidase $A$, composed of one $\alpha$ and one $\beta$ subunit, and $\beta$ hexosaminidase $B$, which contains two $\beta$ subunits. ${ }^{2}$ The gene HEXA encodes the a subunit, and mutations affecting this gene result in a deficiency of $\beta$-hexosaminidase A, clinically known as Tay-Sachs disease. ${ }^{1}$ In Sandhoff's disease, both $\beta$-hexosaminidase A and B have

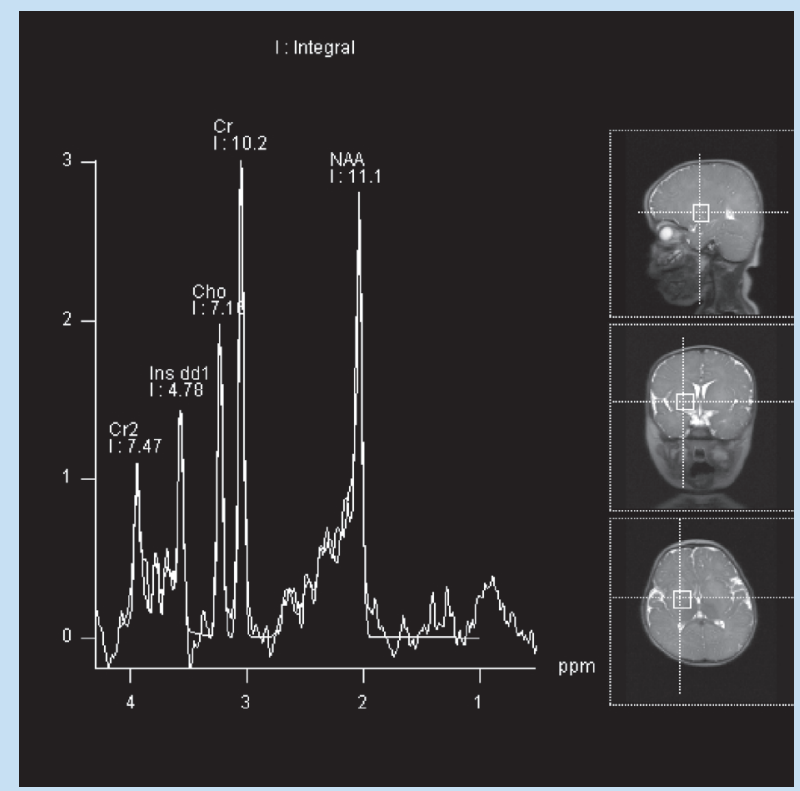

Fig. 4. Magnetic resonance spectroscopy data: single voxel STEAM acquisition (TR/TE $=3000 / 20 \mathrm{~ms}$ ) of the right lentiform nucleus, demonstrating unusual peak configuration near 2.07 ppm.

been found to be deficient secondary to mutations within the HEXB gene which encodes the common $\beta$ subunit. ${ }^{1-3}$ Clinically, these two genetic disorders are indistinguishable from each other. ${ }^{1,4}$

$\beta$-hexosaminidase is a lysosomal enzyme responsible for degrading the ganglioside $\mathrm{GM}_{2}$ to $\mathrm{GM}_{3}$ by removing the $\beta$-N-acetylgalactosamine residue from the nonreducing terminal of the $\mathrm{GM}_{2}$ ganglioside. ${ }^{1,5}$ In Sandhoff's disease, total hexosaminidase activity is reduced to less than $2-3 \%$ of normal, unlike Tay Sachs, where total hexosaminidase activity is preserved due to functional $\beta$ hexosaminidase B activity. ${ }^{5}$ However, the B isoenzyme is unable to hydrolyse the $\mathrm{GM}_{2}$ ganglioside, which accumulates in the brain ${ }^{5}$ and also within the liver, spleen, and kidneys, which may become enlarged. ${ }^{4,5,14}$

Gangliosides have been found to be present primarily within grey matter nuclei and, to a lesser degree, within myelin sheaths of the white matter. ${ }^{6} \mathrm{GM}_{2}$ ganglioside accumulation within lysosomes of cortical neurons results in distension of neuronal cell bodies and nucleus displacement. ${ }^{5,78}$ Over time, this cellular enlargement results in neuronal dysfunction and severe neurodegeneration from eventual neuronal loss. ${ }^{5,8-10}$ Neuronal deterioration and death results in cortical atrophy with widened sulci, narrowed gyri, dilated ventricles and atrophy of the optic nerves and cerebellum. ${ }^{9}$ Ganglioside storage has been found to induce degeneration of white matter. ${ }^{5,10}$ Pathologically, there is evidence for demyelination ${ }^{11,12}$ as well as delayed myelination, ${ }^{11}$ which is believed to be secondary to the grey matter disease. ${ }^{13}$ At autopsy, lipid storage and oedema have been found within the white matter. ${ }^{14}$

While the biochemical basis of Sandhoff's disease is well understood, the exact aetiology resulting in neuronal death and cerebral atrophy is yet to be elucidated..$^{15}$ Animal models have sug- 
gested that neurons distorted by accumulated sphingolipids directly activate inflammatory pathways and cause eventual apoptosis. ${ }^{14,15}$ Sphingolipid accumulation is also thought to incite reactive gliosis, resulting in a progressive increase of head circumference by $1 \frac{1}{2}$ to 2 years of age. ${ }^{12,15-17}$

The clinical expression of Sandhoff's disease is variable. The classic infantile form is characterised by an onset between 3 and 9 months of age, ${ }^{1,16,18}$ following initial normal development. Clinical manifestations are variable and may include initiation of a pronounced startle response after 3 or 4 months of age. ${ }^{17,19}$ Previously acquired developmental milestones decline, and severe muscular hypotonia, followed by spastic quadraparesis and seizures, are additional features. ${ }^{16,18}$ Macular cherry red spots are occasionally observed, ${ }^{17,19}$ though this finding is nonspecific and also seen in several other lysosomal storage disorders. Disease progression is rapid, resulting in death by 3 to 5 years of age. Currently, there is no curative treatment and therapy remains supportive. ${ }^{18}$

The juvenile/subacute variant often presents with ataxia between 2 and 5 years of age, ${ }^{19}$ and has a slowly progressive course and more favourable prognosis. ${ }^{1,2}$ The adult/chronic subtype generally presents in the second or third decade of life, with a variable clinical picture of either spinocerebellar degeneration or motor neurone disease ${ }^{19}$ and typically has a fair survival rate..$^{1,2,19}$

\section{Imaging}

Although Sandhoff's disease has been traditionally considered to be a grey matter disease, neuroimaging studies have characterised abnormalities within both the white and grey matter of Sandhoff patients. Despite extensive variability in findings among studied patients, bilateral symmetric thalamic changes have been found to be an early, predictable finding that is probably specific to the $\mathrm{GM}_{2}$ gangliosidoses, such as Sandhoff's disease, and may be useful in determining whether to perform more specific investigations in infants suspected of having a neurodegenerative disease. ${ }^{5,17}$ The thalami are usually homogeneously hyperdense on computed tomography $(\mathrm{CT})^{5,16,17,20}$ and T2 hypointense ${ }^{11,12,20}$ and T1 hyperintense ${ }^{12,20}$ on magnetic resonance imaging (MRI). MR findings have been found to be particularly variable within the caudate nucleus, globus pallidus, and putamen, though these structures have been shown to be T2-hyperintense. ${ }^{17}$

Currently, there are several theories as to how the stored sphingolipids affect neuroimaging. Abnormal accumulation of macromolecules or lipids increases tissue viscosity and shortens T2 relaxation time, manifesting as a decreased T2 signal, explaining the MR findings among Sandhoff patients. ${ }^{8}$ Dystrophic calcification involving the thalami is thought to be responsible for the hyperdense CT appearance. ${ }^{7,8,17}$ However, it is generally thought that the neuroimaging features are the end result of intralysosomal storage and gliosis owing to axonal and myelin loss in the central cortical neurons. ${ }^{8,16,17}$

The white matter changes in Sandhoff's disease are best appreciated with MRI. During the early, asymptomatic disease stage, normal neurological development ${ }^{20,21}$ and MRI neuroimaging ${ }^{6}$ presume normal myelination. ${ }^{20}$ Later imaging of cerebral white matter has dem- onstrated homogeneous or patchy T2 hyperintensity within frontal, ${ }^{18}$ temporal and occipital regions, ${ }^{6,18}$ and periventricular white matter, ${ }^{18}$ and also within the internal and external capsules. ${ }^{11}$ Involvement of brainstem white matter has been found to extend from the internal capsules to the level of the pons, ${ }^{17}$ medulla, ${ }^{20}$ and cerebellar white matter, ${ }^{20}$ though infratentorial involvement is regarded as secondary to the primary abnormalities of the supratentorial white matter. ${ }^{17}$ These late-occurring white matter abnormalities are presumed to be the result of secondary demyelination following cortical neuronal death and axonal deterioration, rather than from primary dysmyelination. ${ }^{13,20,22}$ However, a combination of delayed myelination and demyelination ${ }^{11,17}$ has also been proposed. Diffuse thinning of the corpus callosum as well as both cerebellar and cerebral cortical atrophy have been demonstrated as abnormal signal intensities on T1- and T2-weighted images that have invariably been attributed to disturbed myelination or calcification., ${ }^{5,10,11,17,20,23}$

A correlation between the degree of thalamic hyperdensity and the presence or severity of white matter disease or atrophy has not yet been demonstrated. ${ }^{5,11,17}$

Proton magnetic resonance spectroscopy (MRS) has revealed progressive elevation in myoinositol $(\mathrm{mI}): \mathrm{Cr} \operatorname{ratios}^{12,18}$ within white matter, grey matter, and the thalamus. ${ }^{18}$ As the $\mathrm{mI}$ peak signifies glial cell activity, it has been hypothesised that accumulation of $\mathrm{GM}_{2}$ gangliosides results in glial cell proliferation, or reactive gliosis. ${ }^{12}$

Demyelination in Sandhoff's disease is expected as a consequence of neuronal loss although, despite clinical and radiographic deterioration, $\mathrm{Cho} / \mathrm{Cr}$ ratios have been found to remain normal. ${ }^{12,18}$ It is thought that the accumulation of gangliosides within cell membranes results in a relative reduction of Cho precursors, thus minimising MRS evidence of demyelination. ${ }^{12}$

NAA and N-acetylaspartylglutamate (tNAA) are markers of neuroaxonal integrity and have been found to decline with neuronal damage and loss. ${ }^{12,25,26}$ Progressive declines in NAA and tNAA, consistent with neuronal loss, have been observed in Sandhoff patients. ${ }^{12,18}$

Sandhoff's disease is characterised by the accumulation of $\mathrm{N}$ acetylhexosamine-containing oligosaccharides, both within glycosphingolipids and as free oligosaccharides, both of which contain $\mathrm{N}$-acetyl moieties. ${ }^{10,26}$ Further analysis has revealed that it is the level of stored glycosphingolipids, and not the level of cerebral oligosaccharides, that correlates with disease severity. ${ }^{10}$ Both animal and human data have confirmed a new spectrum adjacent to the tNAA resonance with a resonance frequency of approximately $2.07 \mathrm{ppm}$ which has been assigned to the $\mathrm{N}$-acetyl moiety of $\mathrm{N}$-acetylhexosamine (NAHex). ${ }^{10,18,26}$ Human data have shown this resonance to be most prominent in white matter and the thalamus and, to a lesser degree, within paramedian parietal grey matter, though this was not seen within the basal ganglia. ${ }^{18}$ This resonance is believed to relate directly to the pathophysiology of Sandhoff's disease by suggesting that oligosaccharides containing NAHex accumulate in cortical and subcortical grey matter as well as in white matter. ${ }^{18}$ Animal proton MRS studies have demonstrated consistently higher intensities of $\mathrm{N}$ acetyl resonances than controls, and have also shown a progression of increasing intensity of these resonances from presymptomatic 


\section{CASE REPORT}

to terminal disease stages. ${ }^{10,26}$ This presymptomatic NAHex resonance has yet to be demonstrated within presymptomatic Sandhoff patients. ${ }^{18}$

Knowledge of the existence of the NAHex resonance may be of direct clinical relevance in the future as the use of MRS broadens to include both non-invasive diagnostic and treatment strategies. ${ }^{10,12}$ This approach may also be helpful in evaluating potential experimental strategies in the treatment of this and other disorders of lysosomal storage ${ }^{10,26}$ as well as in helping to monitor disease progression. ${ }^{12}$

1. Maegawa GHB, Stockley T, Tropak M, et al. The natural history of juvenile or subacute $\mathrm{GM}_{2}$ gangliosidosis 21 new cases and literature review of 134 previously reported. Pediatrics 2006; 118(5): 1550-1562.

2. Zhang Z, Wakamatsu N, Mules EH, et al. Impact of premature stop codons on mRNA levels in infantile Sandhoff disease. Hum Mol Genet 1994; 3(1): 139-145.

3. O'Dowd BF, Klavins MH, Willard HF, et al. Molecular heterogeneity in the infantile and juvenile forms of Sandhoff disease (O-variant $\mathrm{GM}_{2}$ Gangliosidosis). J Biol Chem 1986; 261(27): 12680-12685.

4. Faerber EN, Young Poussaint T. Magnetic resonance of metabolic and degenerative diseases in children. Top Magn Reson Imaging 2002; 13(1): 3-22.

5. Brismar J, Brismar G, Coates R, et al. Increased density of the thalamus on CT scans in patients with $\mathrm{GM}_{2}$ gangliosidoses. Am J Neuroradiol 1990; 11: 125-130.

6. Koelfen W, Freund M, Jaschke W, et al. GM-2 gangliosidosis (Sandhoff's disease): two year follow-up by MRI. Neuroradiology 1994; 36: 152-154

7. Chen C-Y, Zimmerman RA, Lee C-C, et al. Neuroimaging findings in late infantile $\mathrm{GM}_{1}$ gangliosidosis. Am J Neuroradiol 1998; 19: 1628-1630.

8. Autti T, Joensuu R, Aberg L. Decreased T2 signal in the thalami may be a sign of lysosomal storage disease. Neuroradiology 2007; 49: 571-578

9. Becker LE. Lysosomes, peroxisomes and mitochondria: function and disorder. Am J Neuroradiol 1992, 13: 609-620.
10. Lowe JP, Stuckey DJ, Awan FR, et al. MRS reveals additional hexose $\mathrm{N}$-acetyl resonances in the brain of a mouse model for Sandhoff disease. NMR Biomed 2005; 18: 517-526.

11. Alkan A, Kutlu R, Yakinci C, et al. Infantile Sandhoff's disease: multivoxel magnetic resonance spectroscopy findings. J Child Neurol 2003; 18: 425-428.

12. Assadi M, Baseman S, Janson C, et al. Serial $1 \mathrm{H}-\mathrm{MRS}$ in $\mathrm{GM}_{2}$ gangliosidoses. Eur J Pediatr 2007; DOI 10.1007/s00431-007-0469-0.

13. Kroll RA, Pagel MA, Roman-Goldstein $\mathrm{S}$, et al. White matter changes associated with feline $\mathrm{GM}_{2}$ gangliosidosis (Sandhoff disease): Correlation of MR findings with pathologic and ultrastructural abnormalities. Am J Neuroradiol 1995; 16: 1219-1226.

14. Huang J, Trasler JM, Igdoura S, et al. Apoptotic cell death in mouse models of $\mathrm{GM}_{2}$ gangliosidosis and observations on human Tay-Sachs and Sandhoff disease. Hum Mol Genet 1997; 6(11): 1879-1885.

15. Myeorwitz R, Lawson D, Mizukami H, et al. Molecular pathophysiology in Tay-Sachs and Sandhoff diseases as revealed by gene expression profiling. Hum Mol Genet 2002; 11(11): 1343-1350.

16. Caliskan M, Ozmen M, Beck M, et al. Thalamic hyperdensity - is it a diagnostic marker for Sandhoff disease? Brain Dev 1993; 15(5): 387-388.

17. Yuksel A, Yalcinkaya C, Islak C, et al. Neuroimaging of four patients with Sandhoff disease. Pediatr Neurol 1999; 21: 562-565

18. Wilken B, Dechent P, Hanefeld F, et al. Proton MRS of a child with Sandhoff disease reveals elevated brain hexosamine. Eur J Paediat Neurol 2008; 2(1): 56-60.

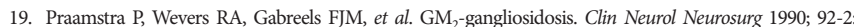
$143-148$

20. Hittmair K, Wimberger D, Bernert G, et al. MRI in a case of Sandhoff's disease. Neuroradiology 1996, 38: S178-S180

21. Schulte FG. Clinical course of $\mathrm{GM}_{2}$ gangliosidoses. A correlative attempt. Neuropediatrics 1984; 15 66-70.

22. Kendall BE. Disorders of lysosomes, peroxisomes, and mitochondria. Am J Neuroradiol 1992; 13: 621653

23. Mugikura S, Takahashi S, Higano S, et al. MR findings in Tay-Sachs Disease. J Comput Assist Tomogr 1996: 20(4): 551-555.

24. Valk J, van der Knapp MS. MR of Myelin, Myelination, and Myelin Disorders, 2nd ed. New York: Springer, 1995: 80-89.

25. Filippi CG, Ulug AM, Deck MDF, et al. Developmental delay in children: assessment with proton MR spectroscopy. Am I Neuroradiol 2002; 23: 882-888.

26. Lowe JP, Stuckey DJ, Awan FR, et al. MRS reveals new resonances in extracts of Sandhoff mouse brain Proc Intl Soc Mag Reson Med 2003; 11: 1957. 